\title{
Know your limits: Machine learning with rejection for vehicle engineering
}

\author{
Kilian Hendrickx ${ }^{1,2[0000-0002-7335-4542]}$, Wannes Meert ${ }^{2[0000-0001-9560-3872]}$, \\ Bram Cornelis ${ }^{1}$, and Jesse Davis ${ }^{2}[0000-0002-3748-9263]$ \\ 1 Siemens Digital Industries Software, Interleuvenlaan 68, 3001 Leuven, Belgium \\ \{kilian.hendrickx, bram. cornelis\}@siemens.com \\ ${ }^{2}$ KU Leuven, Department of Computer Science, Celestijnenlaan 200A box 2402, \\ 3001 Leuven, Belgium \\ $\{$ kilian.hendrickx, wannes.meert, jesse.davis\}@cs.kuleuven. be
}

\begin{abstract}
New vehicle designs need to be tested in representative driving scenarios to evaluate their durability. Because these tests are costly, only a limited number of them can be performed. These have traditionally been selected using rules of thumb, which are not always applicable to modern vehicles. Hence, there is a need to ensure that vehicle tests are aligned with their real-world usage. One possibility for obtaining a broad real-world usage overview is to exploit the data collected by sensors embedded in production vehicles. But these do not produce the detailed data needed to derive the metrics computed using expensive sensors during testing. Therefore it is necessary to correlate the low-end sensor measurements available in production vehicles with the relevant metrics acquired using high-end sensors during testing. Machine learning is a promising avenue for doing this. The key challenge is that vehicles will be used "in the wild" in many scenarios that were not encountered in the controlled testing environment, and it is unlikely that learned models will perform reliably in these previously unseen environments. We overcome this challenge by allowing learned models to abstain from making a prediction when unexpected vehicle usage is identified. We propose a general framework that combines standard machine learning with novelty detection to identify previously unseen situations. We illustrate our framework's potential on data we collected from a large-scale roadroughness analysis use case. Empirically, our approach can identify novel road types in the wild and by doing so it yields better performance.
\end{abstract}

Keywords: Vehicle engineering $\cdot$ Machine learning with rejection $\cdot$ Novelty detection.

\section{Introduction}

When working on new car designs, manufacturers validate that their vehicle meets safety requirements and ensure that its components will be sufficiently durable. These evaluations involve operating the vehicle in a number of development tests, measuring several Key Performance Indicators (KPI) with high-end 
sensors. However, these tests are time-consuming and very costly to carry out as some sensors cost $>\$ 100000$. This precludes carrying out an extensive number of tests and hence requires selecting a small but representative subset corresponding to typical vehicle usage. Traditionally, common real-world usage was deduced from polls and small-scale tests using high-end sensors [10, 34].

Recent developments in connected cars give manufacturers access to a rising amount of real-world vehicle usage data $[14,23,31]$. However, this data is of lower quality than the data from development tests. Unfortunately, it is impossible to directly compute many of the relevant KPIs from the data produced by the low-end sensors included in production vehicles. Traditionally, the relation between the low-end sensor data and high-end sensors KPIs is manually derived using extensive engineering insights and expertise, which is difficult and costly to scale $[28,30]$. Machine learning has been proposed to overcome this challenge, by automatically inferring the relationship between the low and highquality data $[2,22]$. However, this poses a major challenge: learned models are only reliable in situations similar to their training data. In contrast, real-world vehicle usage is not limited to scenarios that arose in the restricted number of development tests. Hence, a model's prediction for a KPI should only be trusted if it derives from a situation that was encountered during the testing phase.

We tackle this challenge by pursuing the recently emerging machine learning paradigm of incorporating a novelty reject option into a model [17]. Such an option permits a model to abstain from making a prediction for samples that substantially differ from those present in the training set. For vehicle usage analysis, using a reject option provides two benefits. First, it enables analyzing real-world vehicle usage data without artifacts arising from unexpected vehicle usage. For example, manufacturers might be interested in the distributions of most common road types customers drive over. A model estimating these types might make incorrect predictions due to encountering unexpected road types. Second, a rejection indicates an unexpected vehicle usage event, which might be accounted for in future vehicle designs and vehicle development tests. For example, the rejects can indicate novel road types in the road analysis use case.

Motivated by vehicle usage analysis, we propose a framework that provides a learned model with a reject option by combining a standard predictive model with a novelty detector. Our approach is model-agnostic in that it works with any predictive model. We evaluate our approach on a novel data set we collected by monitoring a vehicle that was driven over 20000 kilometers over the course of nearly two years. Concretely, we show how incorporating a reject option substantially improves predictive performance when estimating road roughness in the wild. To summarize, we make the following contributions:

1. Highlight the data science challenges that arise when analyzing real-world vehicle usage to inform design engineering;

2. Propose a model-agnostic machine learning with rejection framework that helps overcome some of the aforementioned challenges for this task;

3. Demonstrate the efficacy of our framework on a large-scale road-roughness analysis use-case. 


\section{Background}

\subsection{Vehicle engineering: the need for usage profiling}

Manufacturers need to constantly trade off performance attributes. For example, lightweight designs lead to better fuel efficiency [21], but this needs to be balanced against durability and safety aspects [15]. Instead of relying on rules of thumb for design decisions as was done in the past, pursuing environmentally friendly designs requires aligning performance attributes with real-world usage.

Nowadays, both the design and its requirements can be optimized by better insights into real-world vehicle usage. Firstly, these insights help engineers to better tailor vehicle designs to the targeted customer market. For example, one could adapt vehicles for different geographical markets based on the quality of the roads within each region [11]. Secondly, validating the requirements in development tests is an expensive development phase, as it involves high-end sensors, specialized proving grounds, and skilled drivers [8]. Consequently, insights in real-world usage help to minimize testing time by selecting the most representative scenarios $[13,24]$.

\subsection{Related work on machine learning with a reject option}

Machine learned models have difficulty extrapolating from the data they were trained on. Hence, if a model encounters a sample that is novel or different from what was seen in the training data, it is more prone to making an incorrect prediction [4]. In applications where such mistakes are costly, it might be better to abstain from making a prediction [17]. This option to reject can improve the model's reliability by providing more robustness and better overall performance [27], which ultimately increases the trustworthiness of its predictions [32].

Formally, we can define a machine learning model with a reject option as a function pair: a predictor $h$ and a rejector $r$ [6]. The predictor models the relation $h: X \rightarrow Y$ for the input space $X$ and output space $Y$. The rejector $r: X \rightarrow\{$ accept, reject $\}$ assesses whether or not $h$ is likely to produce an incorrect prediction (reject) or not (accept) for a given sample. This work focuses on novelty rejection, where the goal is to abstain from making a prediction if a test sample is too dissimilar to the training data. This setting is fundamentally different than ambiguity rejection, for example studied in [7], which aims to abstain from making a prediction for samples that fall in a region of the instancespace where $h$ was unable to find a deterministic relation between the input variables and the target.

Ideally, the rejector would decide to abstain from making a prediction based on the predictor's epistemic uncertainty, indicating its lack of knowledge [19]. When a prediction for a sample has high epistemic uncertainty, this indicates that the prediction is likely incorrect. However, truly quantifying this uncertainty is hard. Instead, most rejection approaches either evaluate the predictor's output or directly embed the rejector into the predictor. The former uses soft-predictions, for example provided by a Neural Network's activation layer [5], or exploits 
specific model properties such as a sample's distance to a point of interest $[9$, 35]. Both are assumed to monotonically map the uncertainty. The latter embeds the rejector into the predictor to combine both tasks into a single model, for example by considering multiple one-class models [16] or designing a Neural Network with two output layers [33]. However, this requires $h$ to simultaneously solve its primary task of inferring $X \rightarrow Y$ as well as to faithfully modeling the training data.

Both approaches have rejectors tied to a specific class of models, conflicting with our aim of developing a general framework, applicable for various classification and regression tasks. Instead, we simply assume that the predictor is likely to be inaccurate on data points that are highly dissimilar to those samples in the training data. This yields a model-agnostic approach where $r$ models the training data using, for example, a one-class model such as a Gaussian-mixture [20] or a One-Class Support Vector Machine (OCSVM) [3,18]. During deployment, $r$ only passes samples to $h$ that are similar to those found in the training data.

Regression with a novelty reject option is extremely rare $[17,7]$. Our machine learning contribution is that we study a model-agnostic reject option for regression problems.

\section{Usage profiling: data science challenge}

The vehicle usage profiling task involves deriving insights into the real-world usage of production vehicles by the customers that bought the vehicle. The goal is to improve the vehicle's design by better aligning its requirements to how the vehicle will be used in practice. Performing a proper analysis requires taking the following challenges into account:

1. Automatization The same framework should be generic enough that it can be applied to analyze a large number of different usage parameters with minimal modifications. That is, given a new KPI, the time needed to derive a relation between this KPI and the low-end sensors should be minimized.

2. Small data High-end vehicle measurement data is often limited, as it is expensive to collect. The selected machine learning techniques must be able to cope with a limited amount of training data.

3. Incomplete training data Due to cost and time restrictions associated with testing, manufacturers can only collect ground truth data with high-end sensors for a limited number of real-life usage scenarios. The methodology should be robust to data arising from previously unseen scenarios.

4. Identify unexpected usage Vehicles operated in real life are used in unexpected or novel ways. It is important to automatically identify these scenarios as doing so can yield insights that can improve the designs of future vehicles.

5. Model-agnostic architecture Users need to fully trust the model before using it to make impactful decisions. In addition to the required robustness, users might favor white-box techniques or want to reuse established models. To support any such model, the rejection architecture should not pose any constraints on the predictor. 


\section{Our approach for vehicle usage profiling}

Our goal is to investigate how to automatically detect vehicle usage in the real world that differs from scenarios seen during the development tests in order to minimize errors arising from predictions made during unexpected usage. Formally, this translates to the following data science problem:

Given: Training set $X_{\text {train }}$ with target $Y_{\text {train }}$ collected in a controlled setting. Do: Learn a model $h^{\prime}$ that 1 ) predicts $Y$ for samples similar to $X_{\text {train }}$ and 2) abstains from making a prediction for samples highly dissimiliar to $X_{\text {train }}$. The overall goal can be divided into three subtasks: (i) learning a predictive model capturing the relationship between the features and the target variable, (ii) learning a rejector modeling $X_{\text {train }}$ in order to assess how novel a test sample is, and (iii) integrating the rejector's output in order to decide if a prediction should be made for a sample. Next, we describe each component in detail.

\subsection{Predictor $h$}

The predictor models the relationship between the low-end sensors and the desired KPI. This corresponds to the standard machine learning setting, with the model learned from the vehicle development test data $X_{\text {train }}$. As our architecture is model-agnostic, it does not pose any constraints on the considered model class. Moreover, it does not require altering the underlying learning algorithm.

\subsection{Rejector $r$}

The rejector uses a novelty detector to model $X_{\text {train }}$, assessing whether a sample is an outlier to the samples in $X_{\text {train }}$ [26]. We generalize prior work on modelagnostic rejection approaches in the context of classification $[18,20]$, to define four criteria that a novelty detector must satisfy to be useful in the context of vehicle usage:

1. KPIs in vehicle engineering can be discrete or continuous, so the architecture must be applicable to both classification as regression problems. For regression, estimating parameters of novelty detectors such as the expected number of classes or clusters is challenging. Therefore, the novelty detector should require little apriori knowledge about the data distributions.

2. The detector should return a novelty score instead of a hard prediction. This allows a user to control how sensitive the rejector is to novel data points. For example, this allows us to still make predictions for samples that fall just outside a OCSVM's minimal sphere.

3. Novelty scores should be representative of how similar a sample is to the training data. That is, the further a point is from the training set, the higher its novelty. If this were not the case, it would not be possible to tune the rejector's sensitivity by varying its threshold.

4. The model should be able to return scores falling outside the range of scores assigned to samples in the training set. If this were not the case, a novel sample cannot be distinguished from the training data. 
Two models satisfying these criteria for novel samples are k-Nearest Neighbors outlier detector (kNNO) and the OCSVM. kNNO considers test samples that fall in low data density regions to be novel, which is a valid indicator of novelty. OCSVM has been extensively used, and it models the data by finding a hypersphere enclosing the training data.

Novelty detection typically identifies samples that are novel due to differences in the observed value of any feature. However, for this application, we mostly care about differences in features that are most important for the predictor. That is, we do not care if a sample is novel because it arises due to the car being driven on an unusually hot or cold day. Therefore, we restrict the novelty detectors to only use the predictor's most informative features. Moreover, this is beneficial as kNNO struggles with irrelevant features and high-dimensional spaces.

\subsection{Combined model $h^{\prime}$}

Given a test sample $x$, it is necessary to integrate the rejector and predictor to arrive at a final model. Our approach uses the following rule to determine whether to make a prediction for $x$ :

$$
h^{\prime}(x)= \begin{cases}\emptyset & \text { if } r\left(x \mid X_{\text {train }}\right)>t h r \\ h(x) & \text { otherwise }\end{cases}
$$

That is, it first applies the rejector to obtain the novelty score $r(x)$. If $r(x)$ is greater than a user-defined threshold $t h r$, then $h^{\prime}$ abstains from making a prediction. Otherwise, it applies the predictor $h$ to $x$ and returns its prediction. The key parameter is $t h r$ which determines $h^{\prime}$ 's coverage $\left(N_{\text {accepted }} / N\right)$, which is the fraction of test samples for which a prediction is made $\left(N_{\text {accepted }}\right)$ over the total number of samples tested $(N)$. The value of $t h r$ controls the trade-off between $h^{\prime}$ 's predictive performance and its coverage. Lower values of $t h r$ mean that $h^{\prime}$ is very conservative and will only make predictions for samples that are very similar to the training data. Hence, one would expect a higher accuracy on those samples where a prediction was made, but at the cost of abstaining from making a prediction for many test samples. In contrast, picking a larger value for $t h r$ means $h^{\prime}$ will be making predictions for a greater percentage of the test samples, but it comes with an increased risk of making a larger mistake for those samples that differ from the training data.

\section{Use-case: road-roughness analysis}

We will evaluate our rejection-based machine learning methodology by analyzing real-world car usage data that we collected. Specifically, we will focus on assessing road roughness based on the inexpensive sensors that are present in most modern cars. Understanding road conditions is crucial for design as a road's roughness is one of the primary sources of vibrations, which have a large impact in terms of degrading the components in a car. Because geographic differences 
exist, obtaining large-scale insights into the prevalence of different road types and their quality would enable a manufacturer to tailor a new vehicle to the expected conditions in its target market. The gold standard for assessing road roughness is the International Roughness Index (IRI) [29]. Unfortunately, the IRI must be computed using high-end laser sensors [29] which are impossible to include in production vehicles because they are too fragile and expensive. However, validating our methodology requires estimating the road-roughness for the real-world usage data. Since a laser system cannot be used for long-term measurements, we opt for a road roughness KPI derived from a temporarily installed high-end accelerometer [1]. Typically, it is only feasible to use such a sensor in development tests or a low-end end variant of luxury cars with active suspension systems. For the majority of the cars, this is not the case.

We hypothesize that:

1. using the low-end sensors typically included in production vehicles will permit accurately assessing the road roughness;

2. incorporating a rejector will result in better performance than just using a predictor while still maintaining acceptable coverage; and

3. analyzing the rejected segments will give insights into novel road types encountered during daily vehicle usage.

\subsection{Data Collection and Preprocessing}

We collected two datasets using the same vehicle, a controlled data set and a real-world data set. The former contains ground truth data obtained by driving at predefined speeds over Belgium's three most common road types: smooth asphalt, rough asphalt, and concrete slabs. Additionally, we also measured the car while it was idling. Table 1 shows the details about the controlled data along with the observed road roughness for each road type. The real-world data set contains data arising from natural driving behavior collected over a period of 23 months. During this period, the vehicle was used for daily travel by 39 volunteer drivers for over 20000 kilometers, mainly on Belgian roads. To anonymize the data, we did not save driver names and removed data points within a radius of $400 \mathrm{~m}+\operatorname{rand}(100 \mathrm{~m}, 200 \mathrm{~m})$ of each trip's start and end locations.

In both settings, the same data was measured. First, a high-end DC accelerometer $^{3}$ was mounted on the front wheel hub (Figure 1) and sampled at $2048 \mathrm{~Hz}$ to derive the true road-roughness. To remove the low-frequent sensor drift due to the data acquisition system, we used a high-pass filter of $1 \mathrm{~Hz}$. Second, 16 low-end sensors were measured through the vehicle's Controller Area Network (CAN) bus with a sample-and-hold approach at $200 \mathrm{~Hz}$. Table 2 provides a full overview of the sensors and their quantities. Finally, we acquired the vehicle's location through a GPS antenna for documentation purposes.

We process the high-end accelerometer data and low-end control system data in consecutive, non-overlapping, 1-second windows. In each window, we derive the true road-roughness from the high-end accelerometer and extract

\footnotetext{
${ }^{3}$ PCB 3713B $11200 \mathrm{G}$
} 
the 21 statistical features listed in Table 3 for each of the 15 low-end sensors, which yields 315 features. Each feature is normalized using an outlier-robust scaler $\left(X-P_{50 \%}\left(X_{\text {train }}\right) / P_{75 \%}\left(X_{\text {train }}\right)-P_{25 \%}\left(X_{\text {train }}\right)\right)$, where $P_{X}$ corresponds to the $X$ 'th percentile.

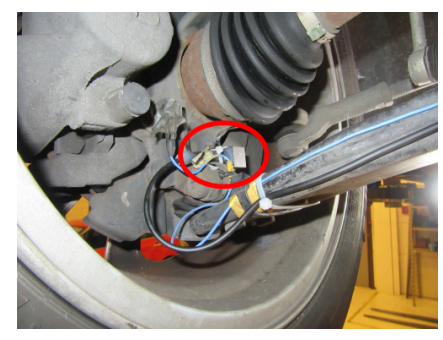

Fig. 1: We instrumented the front wheel hub with a high-end accelerometer in order to derive the true road-roughness.

Table 1: The predefined speeds and road types the controlled data set was collected on. For each category, we indicate its size by total measurement time and indicate its 95 -th percentile $\left(P_{95 \%}\right)$ of the true road roughness.

\begin{tabular}{llcr}
\hline Scenario & Vehicle speeds $(\mathbf{k m} / \mathbf{h})$ & Time $(\mathbf{s})$ & $P_{95 \%}($ Road roughness $)$ \\
\hline \hline Smooth asphalt & $30,40, \ldots, 100$ & 467 & 0.047 \\
Rough asphalt & $30,40, \ldots, 100$ & 386 & 0.190 \\
Concrete & $30,40,50$ & 272 & 0.334 \\
Car idle & 0 & 415 & 0.002 \\
\hline
\end{tabular}

\subsection{Experimental Methodology}

The predictor is a regressor for road-roughness estimation. The rejector is a novelty detector that indicates when to abstain from predicting the roughness. Both algorithms are trained on the data collected in the controlled setting. We evaluate performance on the controlled data using ten-fold cross-validation.

Predictor On the controlled data set we evaluated six different regression models as shown in Table 4. Since this work's main focus is on the rejection component, we opt for the predictor's default parameters as selected in Scikit Learn [25]. shows. For the real-world analysis, we focus on the two best performing models: Random Forest (RF) and XGBoost. 
Table 2: The 15 low-end sensors used to estimate the road-roughness

\begin{tabular}{llll}
\hline Sensor & Unit & Sensor & Unit \\
\hline \hline Vehicle speed & $\mathrm{Km} / \mathrm{h}$ & Total vehicle acceleration & $\mathrm{m} / \mathrm{s}^{2}$ \\
Yaw & $\mathrm{Rpm}$ & Low-end accelerometer & Unknown \\
Wheel propulsion torque & $\mathrm{Nm}$ & Gear indicator & Gear \\
Angle steering wheel & Degrees & Rotational speed front right wheel Rpm \\
Tortion bar torque & $\mathrm{Nm}$ & Rotational speed front left wheel & $\mathrm{Rpm}$ \\
Speed limit & Unknown & Rotational speed rear right wheel & $\mathrm{Rpm}$ \\
Lateral accelerations & $\mathrm{m} / \mathrm{s}^{2}$ & Rotational speed rear left wheel & $\mathrm{Rpm}$ \\
Longitudinal accelerations $\mathrm{m} / \mathrm{s}^{2}$ & & \\
\hline
\end{tabular}

Table 3: The 21 statistical features extracted from each of the low-end sensors

\begin{tabular}{|c|c|c|c|}
\hline Feature & Formula & Feature & Formula \\
\hline Amplitude & $\max (X)-\min (X)$ & Maximum & $\max (X)$ \\
\hline Crest factor & $\frac{\max (|X-\bar{X}|)}{r m s(X)}$ & Extremum & $\max (|X|)$ \\
\hline Extreme deviation & $\max \left(\frac{\max (X)-\bar{X}}{\operatorname{std}(X)}, \frac{\bar{X}-\min (X)}{\operatorname{std}(X)}\right)$ & Median & $\tilde{X}$ \\
\hline Integration & $\sum_{i=1}^{n} \frac{f\left(x_{i-1}\right)+f\left(x_{i}\right)}{2} \Delta x_{i}$ with $\Delta x_{i}=1$ & Minimum & $\min (X)$ \\
\hline Kurtosis & $\overline{\frac{(X-\bar{X})^{4}}{\operatorname{std}(X)^{4}}}$ & Percentile 10 & $P_{10 \%}(X)$ \\
\hline Markov regression & $\begin{array}{c}\frac{R(1)}{R(0)} \text { with } R(0)=\sum_{i=0}^{n-1} x_{i}^{2} \\
\text { and } R(1)=\sum_{i=0}^{n-2}\left(x_{i} x_{i+1}\right)+x_{0} x_{n-1}\end{array}$ & Percentile 25 & $P_{25 \%}(X)$ \\
\hline Mean & $\bar{X}=\frac{1}{n} \sum_{i=1}^{n} x_{1}$ & Percentile 75 & $P_{75 \%}(X)$ \\
\hline Skewness & $\frac{m_{3}}{m_{2}^{\frac{3}{2}}}$ with $m_{j}=(\overline{X-\bar{X}})^{j}$ & Percentile 90 & $P_{90 \%}(X)$ \\
\hline Standard deviation & $\sqrt{\operatorname{variance}(X)}$ & $\begin{array}{l}\text { RMS } \\
\text { Avg. abs. }\end{array}$ & $\sqrt{\overline{X^{2}}}$ \\
\hline Sum & $\sum_{i=1}^{n} x_{i}$ & deviation & $\overline{|X-\bar{X}|}$ \\
\hline Variance & $\overline{|X-\bar{X}|^{2}}$ & & \\
\hline
\end{tabular}

Table 4: The performance of six different regression models on the controlled data set as determined by ten fold cross validation.

\begin{tabular}{lrcr}
\hline Predictor & \multicolumn{2}{c}{$R^{2}$ Mean Absolute Error Mean Squared Error } \\
\hline \hline Random Forest & 0.5627 & 0.0258 & 0.0028 \\
XGBoost & 0.5435 & 0.0304 & 0.0029 \\
Regression Tree & 0.2800 & 0.0338 & 0.0046 \\
k-Nearest Neighbors Regressor & -0.0203 & 0.0506 & 0.0065 \\
Linear Regression & -0.1287 & 0.0612 & 0.0072 \\
Support Vector Regressor & -0.2429 & 0.0717 & 0.0079 \\
\hline
\end{tabular}


Rejector We study two types of novelty detectors that require limited a-priori knowledge about the data distributions:

1. OCSVM, which is a domain-based method that attempts to model the training data's boundary. We use a RBF kernel with $\nu=0.005$ and $\gamma=0.1$.

2. $\mathrm{kNNO}$, which is a distance-based method that attempts to capture the training set's density. We set $k=10$ and use the Euclidean distance.

To limit the dimensionality of data, the rejector only considers the chosen predictor's 10 top-ranked features. These were determined using the Gini importance metric for RF and the average gain across all splits the feature for XGBoost.

\subsection{Results}

Figure 2 shows how the Mean Absolute Error (MAE) varies as a function of coverage on the real-world data. We compare each predictor/rejector model with a standard learned model that makes a prediction for every sample. Additionally, for the predictor/rejector model, we show the MAE on the rejected samples to provides insights into the relationship between novelty scores and prediction errors.

Hypothesis 1: Low-end sensors permit accessing the road roughness.

The standard model that always makes a prediction obtains a MAE of around 0.05 for both XGBoost and RF. Given that the road roughness scores lie between 0 and 0.334 in the controlled data, this performance is sufficient to distinguish among the most common road types as well as capture variation within a single road type.

Hypothesis 2: Incorporating a rejector results in better performance, while maintaining an acceptable coverage.

The results show that our proposed predictor/rejector combinations outperform the standard learned model that always makes a prediction. This holds even when they only abstain from making a prediction for a relatively small fraction of the data. In aggregate, (much) larger errors are made on the rejected samples. For example, without making claims about the model's optimal coverage which is application dependent, Table 5 shows that abstaining from making a prediction on the top $5 \%$ most novel samples reduces the model's MAE by between $10 \%$ and $15 \%$. The MAE on the rejected samples is up to $275 \%$ higher than the MAE on the standard learned model. Our approach is also more robust as it yields lower variances on the prediction errors. Compared to the standard model, this reduces the variance by up to $53 \%$. The improved performance and robustness will increase users' trust and confidence in the model.

For high coverages, using the OCSVM as the novelty detector outperforms using kNNO. However, its advantage diminishes for lower coverages. This is because OCSVM novelty scores are not informative when a test sample is very similar to the training data as these points fall within the hypersphere that the OCSVM uses to model the training data. 

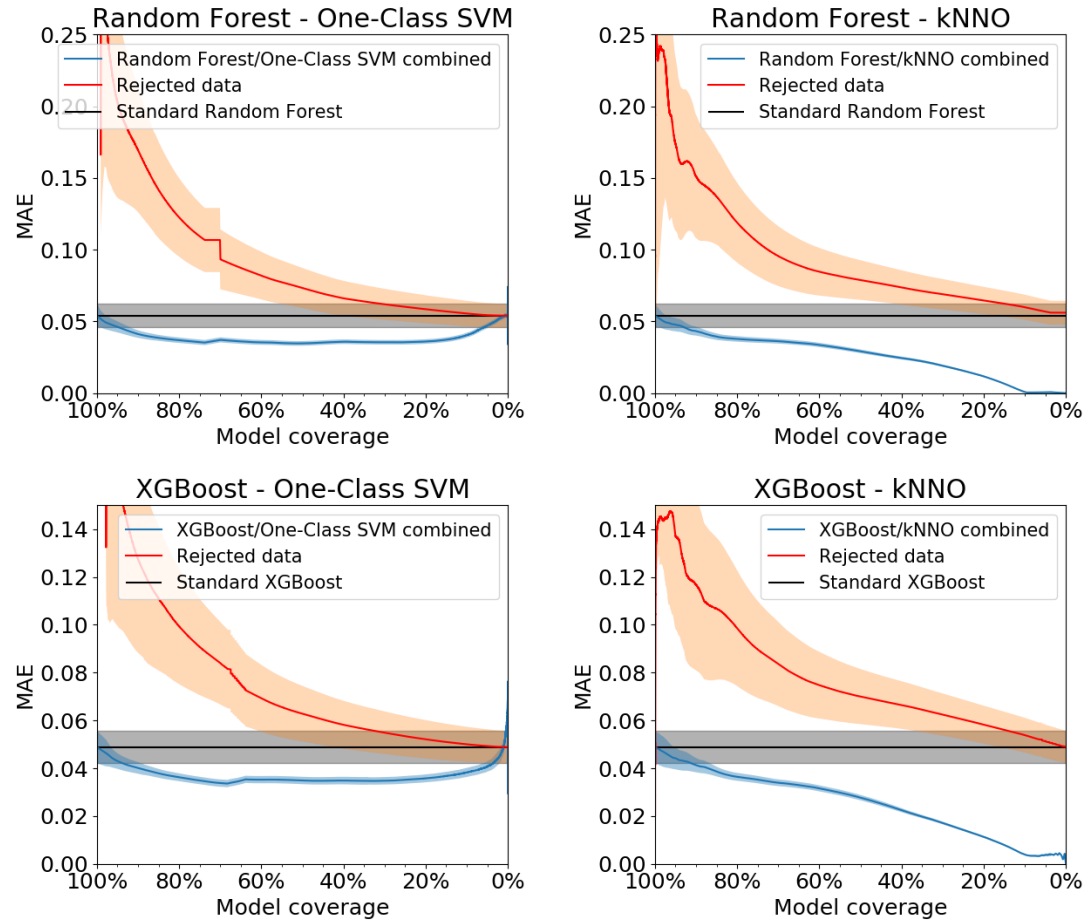

Fig. 2: Each plot shows the MAE (solid line) and error variance (band around MAE) of a standard model that always makes a prediction (black) and a specific predictor/rejector combination (blue) as a function of coverage. The combined predictor/rejector model always outperforms the standard model. Evaluating the MAE of rejected samples (red) shows that the model makes bigger mistakes on the most novel samples, implying a relationship between the rejector's novelty score and the magnitude of the error in a prediction.

Table 5: The percent change in MAE and variance of the combined predictor/rejector models for $95 \%$ coverage relative to the standard model. By rejecting samples that tend to yield high prediction errors, the predictor/reject model yields both better performance and robustness, thereby increasing trust.

\begin{tabular}{ll|ll|ll}
\hline & Predictor & \multicolumn{2}{c|}{ RF } & \multicolumn{2}{c}{ XGBoost } \\
& Rejector & kNNO & OCSVM & kNNO & OCSVM \\
\hline \hline \multirow{2}{*}{ Accepted data } & MAE & $-11.7 \%$ & $-14.5 \%$ & $-9.9 \%$ & $-12.0 \%$ \\
Variance & $-43.4 \%$ & $-52.6 \%$ & $-31.2 \%$ & $-52.0 \%$ \\
Rejected data & MAE & $+221.7 \%$ & $+275.4 \%$ & $+180.7 \%$ & $+228.0 \%$ \\
\hline
\end{tabular}


Hypothesis 3: analyzing the rejected segments gives insights into novel road types.

A high novelty score indicates a road type that has a different relationship between the measurements from the low-end sensors and the road roughness than was observed in the training data. Indeed, inspecting the road types that receive high novelty scores reveals that these differ substantially from the road types included in the controlled data test. For example, within the top 1\% most novel samples, we observe several novel road types such as those shown in Figure 3.

The MAE on the rejected samples shows that there is a correlation between the magnitude of the predictor's errors and the novelty scores. Larger errors are made on those samples with the highest novelty scores. Lowering the model's coverage monotonically decreases the MAE on the rejected samples. Note that there is high variability in the MAE for coverages close to $100 \%$ as only very few samples are rejected in this region.

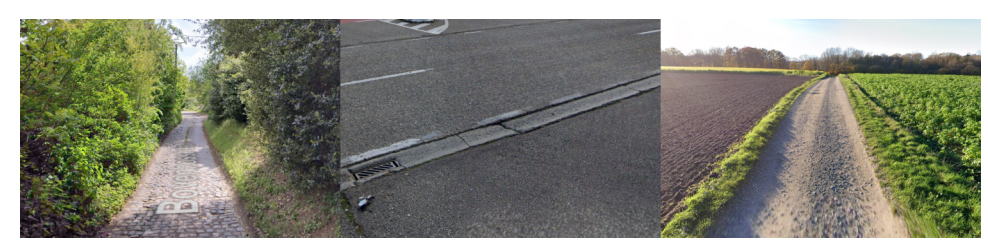

Fig. 3: Analyzing the samples identified as being most novel shows that our methodology finds novel road types. Our results highlight road types such as cobblestones (left [12]), gravel roads (middle [12]), and road gutters (right [12]).

\subsection{Discussion and Lessons Learned}

Our approach offers the capability to abstain from estimating the roughness in novel road conditions, which has three benefits to such analysis. First, automatically detecting and declining to make predictions on samples that deviate from the controlled data yields a more robust and better-performing machine learning model. Consequently, this leads to better insights about expected vehicle usage. If such insights are more accurate and reliable, this results in better vehicle designs and development tests. Second, analyzing the rejected samples offers additional insights, such as identifying road types that are not currently considered in vehicle tests. If a novel road type is frequently encountered in the real-world scenario, this indicates it could be useful to include it in future testing protocols. As we find novelty scores are correlated with the estimation errors, it is easy to prioritize analyzing novel road types based on their prevalence.

More generally, we highlight the lessons learned from this use case:

- Do not underestimate the challenges of collecting real data. First, even for a vehicle shared by multiple drivers, the data set needs to be adequately anonymized such that it does not include any personal information. 
Second, a long-term measurement setup requires regular maintenance and data integrity checks. For example, these checks allowed us to quickly identify issues such as cables being bit by a marten. Interestingly, our methodology assigned high novelty scores to samples where the vehicle's control system produced incorrect measurements, which allowed us to identify the issue and clean the data set.

- Domain knowledge is crucial. While it is appealing to design a fully automated analysis pipeline with machine learning, we found that is generally better to combine automation with engineering insights. First, automated feature selection identified the fuel level indicator sensor as being highly predictive of the road-roughness estimate. Human reasoning helped to identify this as a quirk of the data collection process. The controlled data was collected sequentially per road type without refueling. Second, understanding the sensors allowed us to identify and optimally remove the drift in the measurements of the high-end accelerometer without losing informative data.

- Models with a reject option have significant potential for use-cases where trust is important. Rejection provides more context and transparency about how the learned model will behave as the user will know that the model will abstain from making predictions in novel scenarios. For example, this technology can aid autonomous driving systems by allowing them to return control to the driver by recognizing atypical or previously unencountered scenarios.

\section{Conclusion}

Machine learning with rejection helps to overcome several challenges in vehicle engineering. Enabling machine learning usage to estimate KPIs facilitates scalable usage profiling setups, requiring less or no high-end sensors, and scalable usage profiling analysis, requiring less engineering effort. Additionally, our methodology is more robust to novel vehicle usage, which can enhance the users' trust in the data-driven decision-making process. Finally, its capability to identify novel vehicle usage helps to better align production vehicles with the customer's expectations and actual usage: insights derived from this identification can result in new vehicle requirements or design changes. To summarize, these benefits give better insights into vehicle usage, which helps a manufacturer to optimally design and validate new vehicle models.

We validated our methodology in a road-roughness analysis study. Our results show that this approach returns more robust and accurate estimates of the road-roughness KPI. Interestingly, rejecting only a small fraction of the data already results in drastic improvements, we find a clear relationship between the rejector's novelty score and the roughness estimation error.

\section{Acknowledgments}

KH is funded by VLAIO (Flemish Innovation \& Entrepreneurship) through the Baekeland Ph.D. mandate [nr. HBC.2017.0226]. JD is partially supported by the 
KU Leuven research funds [C14/17/070]. JD and WM received funding from the Flemish Government under the "Onderzoeksprogramma Artificiële Intelligentie (AI) Vlaanderen" programme. The authors have no conflict of interest to declare.

\section{References}

1. Alessandroni, G., Klopfenstein, L.C., Delpriori, S., Dromedari, M., Luchetti, G., Paolini, B.D., Seraghiti, A., Lattanzi, E., Freschi, V., Carini, A., Bogliolo, A.: SmartRoadSense: Collaborative road surface condition monitoring. In: UBICOMM 2014. pp. 210-215. Rome, Italy (2014)

2. Balakrishnan, S., PP, A., Kharul, R.V., C, S.: Accurate Estimation of Time Histories for Improved Durability Prediction Using Artificial Neural Networks. In: SAE Technical Paper (apr 2012)

3. Coenen, L., Abdullah, A.K., Guns, T.: Probability of default estimation, with a reject option. In: 7th Int. Conf. on Data Science and Advanced Analytics (DSAA). pp. 439-448 (oct 2020)

4. Cordella, L., De Stefano, C., Sansone, C., Vento, M.: An adaptive reject option for LVQ classifiers. In: Lecture Notes in Computer Science, vol. 974, pp. 68-73 (1995)

5. Cordella, L., De Stefano, C., Tortorella, F., Vento, M.: A method for improving classification reliability of multilayer perceptrons. IEEE Transactions on Neural Networks 6(5), 1140-1147 (1995)

6. Cortes, C., DeSalvo, G., Mohri, M.: Learning with Rejection. In: 27th Int. Conf. on Algorithmic Learning Theory (ALT). Bari, Italy (2016)

7. Denis, C., Hebiri, M., Zaoui, A.: Regression with reject option and application to kNN. In: Advances in Neural Information Processing Systems (2020)

8. Dodds Colin, J.: Structural testing of complete vehicles, aggregates and components in the laboratory, The Test Engineer's Handbook. 2 edn. (2012)

9. Dübuisson, B., Usai, M., Malvache, P.: Computer aided system diagnostic with an incomplete learning set. Progress in Nuclear Energy 15(C), 875-880 (jan 1985)

10. G. Willén, R., Großkopf, M., Streicher, S., Allouch, R., Heim, T.M.: Fokus Internationalisierung: Herausforderungen in der Definition der Lastdaten für Nutzfahrzeuge. In: Tagung des DVM-Arbeitskreises Betriebsfestigkeit. Ingolstadt, Germany (2014)

11. Gnamm, J., Lundgren, J., Stricker, K., Nilvall, M.: Winning in Europe: Truck strategies for the next decade (sep 2012)

12. Google Street View:

13. Halfpenny, A.: Methods for accelerating dynamic durability tests. In: 9th Int. Conf. on Recent Advances in Structural Dynamics. Southampton, UK (2006)

14. Hamid, A.F.A., Rahman, M.T.A., Khan, S.F., Adom, A.H., Rahim, M.A., Rahim, N.A., Ismail, M.H.N., Norizan, A.: Connected car: Engines diagnostic via Internet of Things (IoT). Journal of Physics: Conf. Series 908(1) (oct 2017)

15. Hammer, B., Klem, A.: Fahrzeugauslegung als Spagat zwischen Leichtbau und Produkthaftung. In: Tagung des DVM-Arbeitskreises Betriebsfestigkeit. Ingolstadt, Germany (2014)

16. Hanczar, B., Sebag, M.: Combination of one-class support vector machines for classification with reject option. Lecture Notes in Computer Science $\mathbf{8 7 2 4}$ LNAI(PART 1), 547-562 (2014)

17. Hendrickx, K., Perini, L., Van der Plas, D., Meert, W., Davis, J.: Machine Learning with a Reject Option: A survey (2021), http://arxiv.org/abs/2107.11277 
18. Homenda, W., Luckner, M., Pedrycz, W.: Classification with rejection based on various SVM techniques. In: Int. Joint Conf. on Neural Networks (IJCNN). pp. 3480-3487 (jul 2014)

19. Hüllermeier, E., Waegeman, W.: Aleatoric and epistemic uncertainty in machine learning: an introduction to concepts and methods. Machine Learning 110(3), 457-506 (mar 2021)

20. Landgrebe, T.C., Tax, D.M., Paclík, P., Duin, R.P., Andrew, C.: A combining strategy for ill-defined problems. In: 15th Annual Symposium of the Pattern Recognition Association of South Africa. pp. 57-62 (2004)

21. Ludanek, H., Bremer, G.: Herausforderungen an die Betriebsfestigkeit zukünftiger Automobilentwicklungen. In: Tagung des DVM-Arbeitskreises Betriebsfestigkeit. Wolfsburg, Germany (2007)

22. Luo, H., Huang, M., Xiong, W.: Application of a Recurrent Neural Network and Simplified Semianalytical Method for Continuous Strain Histories Estimation. Shock and Vibration 2019, 7289314 (2019)

23. Markets and Markets: Connected Car Market by Service - Global Forecast to 2025. Tech. rep., Markets and Markets (2020)

24. P, P.K., J, P., Palanisamy, K.: Optimization of Proving Ground Durability Test Sequence Based on Relative Damage Spectrum. In: SAE Technical Paper (2018)

25. Pedregosa, F., Weiss, R., Brucher, M., Varoquaux, G., Gramfort, A., Michel, V., Thirion, B., Grisel, O., Blondel, M., Prettenhofer, P., Weiss, R., Dubourg, V., Vanderplas, J., Passos, A., Cournapeau, D., Brucher, M., Perrot, M., Duchesnay, É.: Scikit-learn: Machine Learning in Python. Journal of Machine Learning Research 12(85), 2825-2830 (2011)

26. Pimentel, M.A.F., Clifton, D.A., Clifton, L., Tarassenko, L.: A review of novelty detection. Signal Processing 99, 215-249 (jun 2014)

27. Pudil, P., Novovičova, J., Bláha, S., Kittler, J.: Multistage pattern recognition with reject option. In: In. Conf. on Pattern Recognition. vol. 2, pp. 92-95 (1992)

28. Rupp, A., Masieri, A., Dornbusch, T.: Durability Transfer Concept for the Monitoring of the Load and Stress Conditions on Vehicles. In: Innovative Automotive Technology IAT. pp. 31-39. Bled, Slovenia (2005)

29. Sayers, M.W., Karamihas, S.M.: The little book of profiling. Michigan (1998)

30. Städele, M., Rupp, A., Willén, G., Romann, P., Streicher, M.: Ableitung der Beanspruchungs- und Lastzeitfolgen aus Referenzgrö $\beta$ en bei Langzeitmessungen an Nutzfahrzeugen. In: 41. Tagung des DVM-Arbeitskreises Betriebsfestigkeit. Ingolstadt, Germany (2014)

31. Stricker, K., Wegener, R., Anding, M.: Big Data revolutioniert die Automobilindustrie (2014)

32. Varshney, K.R.: On Mismatched Detection and Safe, Trustworthy Machine Learning. In: 54th Annual Conf. on Information Sciences and Systems (CISS). pp. 20192022. Princeton, NJ, USA (2020)

33. Vasconcelos, G., Fairhurst, M., Bisset, D.: Enhanced reliability of multilayer perceptron networks through controlled pattern rejection. Electronics Letters 29(3), 261 (1993)

34. Winterling, H., Teutsch, R., R. Müller, C.W.: Ableitung Einsatzspezifischer Fahrprogramme für die Betriebsfestigkeitserprobung schwerer Nutzfahrzeuge Beispiel Baustellensegment. In: 41. Tagung des DVM- Arbeitskreises Betriebsfestigkeit. Ingolstadt, Germany (2014)

35. Zou, C., Zheng, E.h., Xu, H.w., Chen, L.: Cost-sensitive Multi-class SVM with Reject Option: A Method for Steam Turbine Generator Fault Diagnosis. Int. Journal of Computer Theory and Engineering 3(1), 77-83 (2011) 